\title{
Nursing supervision for using amiodarone hydrochloride injection to tachyarrhythmia patients
}

\author{
Nana Kong \\ Department of Cardiology, Nanjing General Hospital of Chinese PLA, Nanjing Jiangsu, 210002, \\ China
}

Keywords: Amiodarone hydrochloride, Tachyarrhythmia, Mainline, Nursing supervision.

\begin{abstract}
Objective To analyze the nursing management of amiodarone hydrochloride injection in patients with tachyarrhythmias during intravenous injection. Methods Forty-six patients with tachyarrhythmia treated with amiodarone hydrochloride in our hospital from April 2014 to April 2015 were enrolled in this study. The concentration of the drug and the correct blood vessel were selected. The nursing staff pay close attention to Injection site changes, while giving the appropriate care. The blood pressure, heart rate and complications of the patients before and after treatment were observed and analyzed. Results The heart rate slowed down after intravenous infusion of amiodarone in 3 patients, 2 patients improved after intravenous infusion of isoproterenol, and 1 patient was treated with artificial pacemaker. 2 patients with amiodarone-induced blood pressure decreased; 1 patient in the intravenous infusion of amiodarone after 2 hours, there phlebitis-related symptoms, 2 cases occurred in the use of amiodarone within 12 to 24 hours, 1 case The use of amiodarone appeared after 48 hours, 2 weeks after the patients with phlebitis were restored to normal skin. The systolic blood pressure (SBP), diastolic blood pressure (DBP) and heart rate (HR) of patients after intravenous infusion of amiodarone were significantly lower than those before treatment $(\mathrm{P}<0.05)$. Conclusion Strengthening the nursing management of patients using amiodarone hydrochloride injection can effectively enhance the therapeutic effect, protect the safety of patients and improve their quality of life, should be widely applied in clinical.
\end{abstract}

\section{Foreword}

As one of the common diseases of cardiovascular medicine, tachyarrhythmias have great threat to the life and health of the patients. If the patients do not receive timely treatment or improper treatment, they will endanger life [1]. Treatment of tachyarrhythmias is the most critical step to restore and maintain sinus rhythm. Amiodarone is the main drug used to treat this disease, and amiodarone is effective in improving the prognosis of patients with a high risk of arrhythmia and sudden death. However, the drug also has some adverse reactions, the use of the process must be closely monitored in patients with drug reactions, the implementation of effective patient care management, timely treatment of various types of emergencies, thereby reducing the incidence of complications. Scientific care can improve the safety of treatment, improve the quality of life of patients, improve patient satisfaction with care. In our hospital from April 2014 to April 2015 using amiodarone injection for treatment of 46 patients with arrhythmia care management, access to the ideal therapeutic effect, are as follows.

\section{Information and method}

\section{General information}

46 patients with tachyarrhythmias treated with amiodarone hydrochloride injection from April, 2014 to April, 2015 in our hospital were studied. Among the 46 patients, 24 were female and 22 were male; the age ranged from 40 to 80 years, mean age was (65.68 \pm 12.32 ) years; 12 cases of rheumatic heart disease, 10 cases of coronary heart disease, 8 cases of myocarditis, 3 cases of myocardial infarction, 7 
cases of dilated cardiomyopathy; according to the different types of atrial fibrillation in 14 cases, atrial flutter in 8 cases, 12 cases of ventricular tachycardia, ventricular contraction in 9 cases, 3 cases of supraventricular tachycardia. Thyroid, liver, and kidney function were normal in all subjects, with no symptoms of atrioventricular block or heart failure.

\section{Method}

Intravenous infusion method. In the conventional treatment on the basis of the implementation of patients with amiodarone hydrochloride injection for treatment. In the ECG detection, the infusion concentration to adjust. Nursing staff to follow the doctor's advice, 150mg amiodarone and 20ml concentration of $0.9 \% \mathrm{NaCl}$ for mixing, to be completely dissolved, the patient intravenous injection. At the same time should pay attention to maintain a slow speed, usually in 10 to 15 minutes to end the injection, followed by continuous intravenous infusion. Drip should be based on the weight of patients to determine the load of amiodarone, usually 3mg / kg. Maintained $1 \sim 1.5 \mathrm{mg} / \mathrm{min}, 6$ hours will be $0.5 \sim 1 \mathrm{mg} / \mathrm{min}, 1$ day total of $1200 \mathrm{mg}$. Later can gradually reduce the amount. It is noteworthy that the use of amiodarone intravenous infusion time should not be more than 3 to 4 days in the injection should pay close attention to patients with ECG monitoring information, and ask the patient whether there is discomfort, if there is abnormal situation Should be promptly reported to the attending physician, so as to take timely and effective measures to control the deterioration of the situation.

Observe the patient's complications and the corresponding care. Common complications during IV infusion include bradycardia, decreased blood pressure, and phlebitis. The following are the three kinds of complications that care methods. First, bradycardia is the most common complications, the specific manifestations of chest tightness, dizziness, sinus bradycardia (40 to 50 times / min), nurses of patients with severe arrhythmia intravenous infusion of iodine ketone Should be implemented in patients with real-time monitoring for patients with bradycardia should be in the medication after the heart rate and heart rate were closely observed, the patient's ECG real-time recording. When patients with atrioventricular block or heart rate less than 60 times / min, should immediately stop the medication, and follow the doctor's advice to give patients with isoproterenol or atropine to improve the patient's heart rate, keep the patient's heart rate within the normal range. Some patients need to use amiodarone for long-term treatment, when there bradycardia should be installed artificial cardiac pacemaker, thus ensuring the safety of their medication. Nurses should carefully observe the patient's heart rate, and frequently asked about their feelings, if any abnormalities should promptly inform the doctor to assist them to complete the work and complete the record. Second, blood pressure, arrhythmia more serious patients, the hemodynamics can easily change, treatment should determine whether the decline in blood pressure is due to the primary disease caused by or due to the use of amiodarone. In the intravenous infusion of amiodarone patients should monitor their blood pressure, ECG, the initial phase of blood pressure measurement every $10 \mathrm{~min}$, if patients with stable blood pressure, can be measured once every 30 minutes, then gradually become 30 to 60 minutes once measuring. When the patient's blood pressure should be the implementation of intravenous infusion or pumped dopamine, so that it is more safe and effective treatment. Third, phlebitis. The complications were mainly due to the implementation of amiodarone intravenous infusion caused by chemical phlebitis and acute thrombophlebitis, the higher concentration of amiodarone pumped from the peripheral vein, the patient appears to have the probability of phlebitis is about $88,20 \%$, therefore, in order to reduce the production of phlebitis should be used central venous administration. In summary, the intravenous infusion of amiodarone in patients with effective care should be used to prevent the occurrence of phlebitis.

Intravenous amiodarone in the process, nurses should be closely observed the location of the puncture of the patient, try to use infusion pump to adjust the infusion rate, and drug concentration monitoring. In the vein should be selected on the selection of straight and thick vein infusion, the best of the upper extremity vein, try to avoid the joint parts. Puncture site should be 4 to 6 hours after a replacement, to prevent distal limb swelling. If the patient's puncture site should be rapid replacement 
of swelling, the more severe symptoms should be used in patients with magnesium sulfate injection of local wet compress, 2 times a day for the redness of the symptoms have significantly improved. Nurses should implement strict bed shift regime, before the infusion of $0.9 \% \mathrm{NaCl}$ solution for injection, so that drug remnants of the time in the blood vessels shortened, thereby reducing the vascular wall due to drug stimulation. The patient's skin around the injection site for close observation, when the injection site of patients with burning sensation or pain, even if there is no swelling of the skin should also be the injection site replacement.

Supine, oxygen care. In general, hemodynamic changes occur, the patient will be breathing difficulties, shortness of breath and chest tightness and other symptoms, in order to slow down the body's metabolic rate, reduce myocardial oxygen consumption, should help patients to maintain a high pillow supine, and the doctor's Under the instructions given to patients with oxygen inhalation, to maintain $2 \sim 5 \mathrm{~L} /$ min oxygen flow. Thereby promptly correct the phenomenon of tissue hypoxia, relieve the symptoms of such patients.

Diet and health care. Nurses should be advised to maintain digestible, light diet, while things in the fat content and cholesterol content should be low, avoid spicy spicy food, which will help patients to restore health. Patients with cardiac insufficiency should ensure a low-salt diet. In addition, patients should be informed that attention to the injection site of care, to keep the skin clean and health, attention to the protection of blood vessels.Psychological intervention. Nurses and patients should be active and effective communication, the use of comforting words to psychological counseling to reduce the fear of patients with disease, improve their treatment compliance. In addition, patients should be informed of the disease-related knowledge, so that the correct view of the disease, and master the appropriate means of prevention, to avoid the rapid development of the disease. Patients can also explain the success stories related to enhance their confidence in the treatment.

\section{Efficacy criteria}

The patients' liver, renal function, blood routine, cardiogram and the incidence of adverse reactions were recorded, and the symptoms of the patients at the later stage of treatment were observed and the occurrence of the 24-hour pre-systolic events was observed.

\section{Statistical treatment}

The data were analyzed by SPSS19.0 statistical software, and the data were expressed by ( $\bar{x} \pm s)$, t-test and rate data $(\%)$. The results were analyzed by $\chi^{2}$ test. The results were statistically significant when $\mathrm{P}<0.05$.

\section{Results}

\section{Heart rate and blood pressure before and after medication}

The table 1 shows that patients with amiodarone intravenous drip, the systolic pressure, diastolic blood pressure and heart rate before medication were declined, with significant difference between the data groups $(\mathrm{P}<0.05)$.

\begin{tabular}{cccc}
\multicolumn{4}{c}{ Table 1 Heart rate and blood pressure before and after medication $(\bar{x} \pm s)$} \\
\hline Time & systolic pressure $(\mathrm{mmHg})$ & $\begin{array}{c}\text { diastolic pressure } \\
(\mathrm{mmHg})\end{array}$ & Heart rate $($ times/min $)$ \\
\hline Before medication & $150.32 \pm 10.56$ & $87.85 \pm 9.67$ & $149.67 \pm 20.65$ \\
After medication & $98.50 \pm 7.34$ & $67.43 \pm 8.30$ & $93.46 \pm 11.27$ \\
$\mathrm{t}$ & 9.57 & 4.08 & 5.34 \\
$\mathrm{P}$ & $\mathrm{P}<0.05$ & $\mathrm{P}<0.05$ & $\mathrm{P}<0.05$ \\
\hline
\end{tabular}




\section{Incidence of the disease}

In this study, 3 patients in the use of amiodarone after intravenous infusion of heart rate slowing phenomenon, the intravenous infusion of isoproterenol after 2 patients improved, the other 1 patients with artificial cardiac pacemaker for treatment. 2 patients with amiodarone-induced blood pressure decreased; 1 patient in the intravenous infusion of amiodarone after 2 hours, there phlebitis-related symptoms, 2 cases occurred in the use of amiodarone within 12 to 24 hours, 1 case The use of amiodarone appeared after 48 hours, 2 weeks after the patients with phlebitis were restored to normal skin.

\section{Discussion}

The occurrence of tachyarrhythmia is mainly due to rapid heart pacing in patients with atrial diastolic time is short, leading to blood flow disorders, myocardial ischemia. The disease will cause heavy heart failure in patients [2]. In addition, arrhythmia and other heart disease will occur at the same time, common with coronary heart disease, myocardial infarction, myocardial infarction, so that patients with increased mortality, thus prevention and treatment of arrhythmia imminent. Treatment of arrhythmia at this stage the main use of drug therapy and surgical therapy. Drug therapy is more commonly used as a treatment, and surgical therapy, there are DC transfer, defibrillation and so on. The risk of surgical treatment of large and will bring greater trauma to patients. Therefore, for doctors, the choice of drugs with high safety and efficacy is particularly important. Practical studies have shown that amiodarone in the treatment of arrhythmia has a good effect, the drug can make the action potential of time, refractory period and repolarization time, help to reduce the reentry excited [3]. In addition, amiodarone can block calcium, potassium, sodium channels, and thus inhibit the sodium influx, so that the rate of decline, the autonomy of the sinus node also slowed down to achieve anti-arrhythmic effect . At the same time, amiodarone can arterial dilatation, arterial pressure decreased, the blood flow to play a significant improvement.

At this stage, amiodarone is a broad spectrum of antiarrhythmic drugs, the drug does not exist negative myasthenia gravis, the majority of patients are tolerant of this drug is better, but its lack of room Ventricular node function and sinus node function inhibition, thereby slowing heart rate. In the use of amiodarone should be closely observed in patients with ECG, try to prevent the slow arrhythmia, especially the high degree of atrioventricular block [4]. Patients in the process of intravenous infusion due to vasodilation, rapid infusion of hypotension symptoms occur, which should strengthen the care, close monitoring, thereby reducing the incidence of adverse reactions, thereby enhancing the treatment effect and safe and reliable treatment Sex, so that patients can improve the quality of life. Intravenous amiodarone has the characteristics of rapid, effective and reliable, the drug can significantly improve the risk of sudden death in patients at high risk and the prognosis of arrhythmia patients, thus preventing patients with malignant arrhythmia [5], reduce arrhythmia mortality. Nursing staff should be treated with amiodarone to take care of patients with science, the use of drugs in patients with blood pressure, ECG changes and drug reactions closely observed, while patients with psychological care and symptomatic treatment, thereby reducing complications The incidence of care to improve and reduce the risk of adverse reactions in patients with, enhance the therapeutic effect.

The results of this study showed that three patients in the use of amiodarone after intravenous infusion of heart rate slowing phenomenon, the intravenous infusion of isoproterenol after two patients improved, the other one to install artificial cardiac pacemaker treatment. 2 patients with amiodarone-induced blood pressure decreased; 1 patient in the intravenous infusion of amiodarone after 2 hours, there phlebitis-related symptoms, 2 cases occurred in the use of amiodarone within 12 to 24 hours, 1 case The use of amiodarone appeared after 48 hours, 2 weeks after the patients with phlebitis were restored to normal skin. After the intravenous infusion of amiodarone, the systolic blood pressure, diastolic blood pressure and heart rate of the patients were lower than those of the control group $(\mathrm{P}<0.05)$. Amiodarone can be seen in the treatment of rapid arrhythmia has a more 
significant effect in the intravenous infusion of amiodarone in the course of patients to implement scientific and effective nursing management can reduce the incidence of complications and promote rehabilitation of patients and improve their quality of life , Worthy of promotion.

\section{References}

[1] Wang Dong.Clinical Efficacy and Safety of Amiodarone in Treatment of Patients with Acute Arrhythmia, The latest medical information abstract, 2015, 15 (71) : 24.

[2] Zhao Hui, Liu Qi, Sun Bo. Clinical Study on Treatment of Arrhythmia with Acupuncture and Amiodarone Hydrochloride, Chinese Primary Health Care, 2015, 29 (02) : 112-113.

[3] Hu Zhengdong.Clinical Analysis of Wenxin Granule Combined with Amiodarone Hydrochloride in Treating Chronic Arrhythmia, Modern Traditional Chinese Medicine Journal,2014,8 (17) : 99-100.

[4] Wang Xiaoyou.Prevention of Phlebitis in 100 Cases of Arrhythmia Treated by Amiodarone Hydrochloride, China Pharmaceuticals, 2013,22 (11) : 60-61.

[5] Li Yanjun, Tian Yin, Liu Xia.Nursing Experience of Continuous Intravenous Injection of Amiodarone Hydrochloride Adverse Reaction, Journal of Clinical Medicine, 2016,3 (12) :2335-2336. 